\section{Neoadjuvant treatment of biliary tract cancer: state-of-the-art and new perspectives}

\author{
Marco Imperatori', Loretta D'Onofrio', Eleonora Marrucci', Francesco \\ Pantano', Alice Zoccoli' \& Giuseppe Tonini*,1
}

Gall bladder cancer (GBC), and intrahepatic and extrahepatic (perihilar or distal bile duct's) cholangiocarcinomas (CCA) are usually diagnosed in locally advanced or node-positive stage, with a short survival rate. Thus, it appears essential to explore novel strategies for improving disease downstage and radical surgery. Chemoradiotherapy followed by liver transplantation seems to be one of the most promising approaches for intrahepatic or perihilar disease while chemotherapy with novel radiotherapy techniques (such stereotactic body radiation) emerged as an attractive preoperative treatment in distal diseases. In this paper, we will review currently available knowledge about neoadjuvant treatment of biliary tract cancers (BTC) paying attention to challenges that make this type of management in clinical practice difficult.

Submitted: 10 August 2015; Accepted: 22 October 2015; Published online: 30 November 2015

\section{Practice points}

- Biliary tract cancer (BTC) represents an heterogeneous group of diseases with well established differences in their anatomic origins and biologic behavior. They are relatively chemoresistant and only gemcitabine and cisplatin showed significant efficacy in disease control.

- A radical surgical resection remains the only potentially curative treatment of an early stage BTC.

- Even if attractive, neoadjuvant approach (chemotherapy alone or combined with radiotherapy) cannot be considered the standard of care out of clinical trials.

- A proper selection of BTC patients who may benefit from a neoadjuvant strategy is required, possibly based on: a more accurate stadiation before treatment (such as though PET or explorative laparoscopy); definition of prognostic factors (ECOG PS, markers dosage, age, tumor dimension, etc.); definition of inoperability criteria.

- Chemoradiotherapy followed by orthotopic liver transplantation represents a promising neoadjuvant strategy for stage I and II perihilar-cholangiocarcinoma (CCA) and intrahepatic-CCA, but the presurgery treatment protocol need to be standardized.

- New types of local treatment (such as radioembolization) need to be prospectively studied as conversion treatments in intrahepatic-CCA.

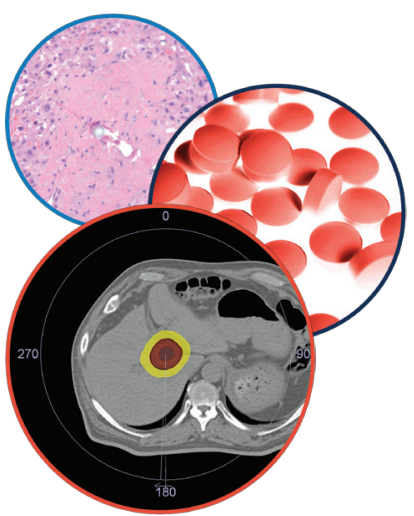

Hepatic Oncology

\section{KEYWORDS}

- biliary tract cancers

- liver transplantation

- loco-regional treatments

- neoadjuvant systemic treatments

'Campus Bio-Medico University of Rome, Rome, Italy 
Biliary tract cancer (BTC) represents a heterogeneous group of tumors deriving from cells lining the biliary tree, including: gall bladder cancer (GBC), and intrahepatic and extrahepatic (perihilar or distal bile duct's) cholangiocarcinomas (CCA) (Figure 1). It remains a group of relatively rare cancers comprising about the $3 \%$ of all gastrointestinal malignancies [1,2]. However, the incidence of CCA appears to be on rise in western populations, particularly, the estimated age-adjusted incidence of intrahepatic CCA (IH-CCA) in the USA increased by $165 \%$ from 1979 to 1999 (from 0.32 per 100,000 in 1975 to 1979 to 0.85 per 100,000 in 1995 to 1999 ) [3] as well as its mortality is increasing $[4,5]$. On the other side, the GBC, which is the most common type of BTC and its most frequent histotype is adenocarcinoma, has an high geographic- and ethnic-dependent variation of prevalence [6] with a recent decline of mortality rates in Europe [7], probably due to changes in risk factors exposure and to an earlier adoption of cholecystectomy.

Therefore, radical surgery remains the only potentially curative treatment of an early stage BTC, but the majority of patients still receive diagnosis of a more advanced stage disease when the tumor is metastatic or locally advanced (with local blood vessels invasion andlor extension to both hepatic lobes or with nodal involvement) [8]. Nevertheless, a number of those patients also present a marginally resectable tumor, but incomplete resections ( $R 1 \backslash R 2)$, even with the addition of postoperative chemotherapy and/or radiation therapy result in outcomes comparable to those of individuals not undergoing surgical resection $[9,10]$.

Preoperative treatment of BTC has always represented a challenging strategy of management of locally advanced or marginally resectable BTC based on the hypothesis that disease downstage before surgery could increase the rates of radical resections (R0) and the longterm survival. However, scientific literature has not reported compelling favorable evidences. In fact, the lack of selection of patients who may benefit from a neoadjuvant therapy facilitating the $\mathrm{R} 0$ resection as well the absence of extensive and prospective studies about this strategy of treatment has meant that neoadjuvant therapy did not represent an accepted standard of care. No guidelines are available nowadays concerning this specific setting of BTC patients and multiple reviews and opinions have concluded by affirming that neoadjuvant therapies of BTC are currently not indicated because of their unproved efficacy and their possible negative effects caused by the delay of a potential curative surgery $[9,11,12]$. In this short review, we present the current evidence about the challenging strategy of preoperative treatment of BTC and its possible future implications in clinical practice.

\section{Methods}

A comprehensive systematic search was performed in MEDLINE database (from 1996 to 2014) using the medical subject headings 'neoadjuvant treatment in intrahepatic cholangiocarcinoma' and 'neoadjuvant therapy in extrahepatic cholangiocarcinoma', 'neoadjuvant treatment in hilar cholangiocarcinoma' or 'neoadjuvant treatment of gall bladder cancer'. We included also data derived from single institution experiences and retrospective studies taking into account the rarity of this type of cancer and the lack of randomized controlled trial.

\section{- Neoadjuvant treatments of gall bladder cancer}

Simple cholecistectomy or cholecistectomy with portal lymphadenectomy (with a resection of a minimum of six nodes) and hepatic resection of the IV and V segment, with or without bile duct excision, are the recommended standard of care of T1a and T1b-T3 GBC, respectively, in order to ensure an $\mathrm{R} 0$ resection [12]. The distant nodes involvement, as well as a high gross primary tumor volume with vascular encasement and lor extensive involvement of the bile duct or other organs represent the criteria of inoperability, where the prognosis of disease is comparable to that of the stage IV GBC.

Neoadjuvant therapy in locally advanced GBC has been mainly explored by several retrospective analysis focusing on its possible effect as conversion therapy and improving survival rates after surgery.

\section{Neoadjuvant chemoradiation}

The majority of scientific data focused on the role of neoadjuvant chemoradiation therapy. One small prospective Chilean study enrolled 23 locally advanced GBC patients to receive neoadjuvant 5-fluorouracil (5-FU)-based chemoradiation. After the preoperative treatment protocol, seven patients were excluded from surgical treatment and 14 patients underwent resection. Treated patients had a worst survival than subjects not treated with presurgery 


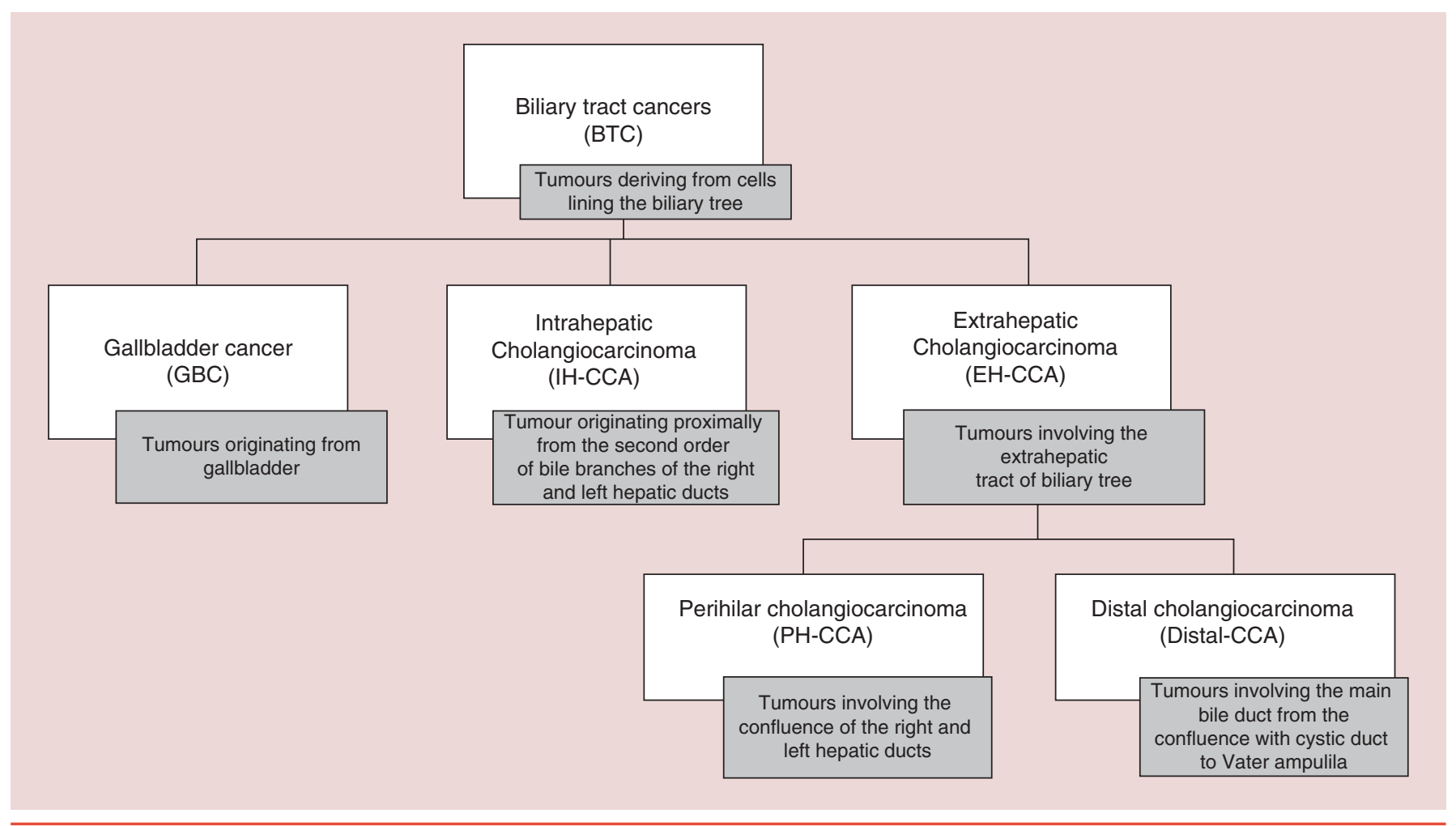

Figure 1. Definition of biliary tract cancers group by anatomic site.

chemoradiation [13]. However, this old study was conducted before the advent of gemcitabinebased chemotherapy in treatment of BTC. Then, the radiation therapy was applied to nonsurgically staged GBC patients, many of whom are found to have peritoneal or nodal disease. On the contrary side, other case reports confirmed preoperative chemoradiation as a potential feasible strategy to improve radical surgery in locally advanced GBC. Engineer et al. [14] obtained two complete metabolic response (on three patients) with IMRT and concomitant gemcitabine, with no or small residual disease at surgery. In this report, all the patients underwent positron emission tomography with computerized tomography scan before and after treatment protocol confirming the early stage at the beginning of chemoradiation.

\section{Neoadjuvant chemotherapy}

The role of the chemotherapy given before surgery has not been deeply explored in recent studies and mixed results have been reported. A retrospective review evaluated the role of adjuvant or neoadjuvant (gemcitabine-based) therapy in treatment of 174 patients affected by $\mathrm{GBC}$ or CCA. The 5-year OS rate after resection of GBC and CCA was 50.6 and $30.4 \%$, respectively. Of those patients, $17.8 \%$ received neoadjuvant chemotherapy. Interestingly, the immediate resection without neoadjuvant chemotherapy was associated with an increased median survival from the date of diagnosis from 42.3 to 53.5 months $(\mathrm{p}=0.01)$. On univariate analysis, but not multivariate analysis, patients who received neoadjuvant therapy had a trend towards increased hazards of death $(\mathrm{HR}=1.66$; 95\% CI: 0.97-2.83; p = 0.07) [15]. On the opposite side, a more recent retrospective analysis evaluated the activity of gemcitabine-platinumbased regimen in 37 GBC patients showing an overall response rate (ORR) of 67.5\% with 17 patients $(46 \%)$ that underwent $\mathrm{R} 0$ resection. The median overall survival (OS) and progression-free survival (PFS) of the whole group was 13.4 and 8.1 months respectively. Patients who underwent surgery had a significantly better OS and PFS (25.8 vs 5.6 months), respectively [16].

\section{- Neoadjuvant treatment of intrahepatic cholangiocarcinoma}

Intrahepatic cholangiocarcinoma (IH-CCA) is the second most frequent type of primary liver cancer, after hepatocellular carcinoma [17] and has worse prognosis comparing to extrahepatic bile duct cancer [18]. In fact, albeit in a locally 
advanced stage, both systemic and local therapies have limited effect in order to arrest tumor growth without any gain of improvements on survival [19-23]. Recently, more retrospective studies reported encouraging results about new local treatments given in order to allow R0 surgery.

\section{Neoadjuvant radioembolization}

Yttrium-90 radioembolization could represent an interesting option as a presurgery and downstaging treatment of non resectable IH-CCA [2426]. In fact, in Mouli's study, 5 of 46 IH-CCA patients previously judged unresectable by a multidisciplinary team were downstaged after Y90 radioembolization; this allowed surgical resection or liver transplantantion. However it is notable that all five patients had PS ECOG zero, solitary peripheral tumors without portal vascular invasion and were treatment naive. Other case reports and small studies described the possibility of an R0 resection of IH-CCA after radioembolization with or without combined chemotherapy [27-29].

\section{Neoadjuvant SBRT}

Stereotactic body radiotherapy (SBRT) has recently emerged as an alternative local ablative therapy. Ibarra et al. [30] showed an overall freedom from local progression (FFLP) of $63 \%$ at a median follow-up of 12.9 months for intrahepatic primary tumor after SBRT. Unfortunately, also hepatocellular carcinoma was included and patient series was very small with only 11 patients affected by BTC.

\section{- Neoadjuvant chemoradiotherapy \& liver transplantation in management of CCA}

One of the most promising strategy of preoperative treatment of IH or perihilar (PH) CCA is the protocol of neoadjuvant chemoradiotherapy followed by orthotopic liver transplantation (OLT). In fact, when there is no metastatic spread of CCA, even with a significant biliary tree involvement preventing the partial liver resection (as it especially occurs in perihilar CCA), the only possibility of a R0 surgery could be the liver transplantation but its utility has remained controversial for many reasons: the lack of organs and the consequent waiting list; the needed immunosuppression accelerating the tumor progression [31,32]. Up to date, several evidences have been reported about feasibility and positive outcome of OLT in management of CCA despite the different kinds of adopted neoadjuvant treatment. In 2002, a single institution study evaluated the effect of neoadjuvant chemoradiation therapy combined with OLT in a selected group of 17 CCA patients (the inclusion criteria were: tumor dimension $<2 \mathrm{~cm}$ without intra- or extra-hepatic disease extension or nodal involvement) treated with neoadjuvant protocol including 60 Gy biliary brachytherapy delivered through percutaneous transhepatic catheters and intravenous infusion of 5 -fluorouracil ( $5 \mathrm{FU}, 300 \mathrm{mg} / \mathrm{m} 2 /$ day) until transplantation. Eleven patients underwent liver transplantation with a median of 3.4 months after diagnosis and 10 of those transplanted patients were affected by histopathologically confirmed PH-CCA. Five of the 11 patients $(45 \%)$ were alive without evidence of tumor recurrence with a median follow up of 7.5 years, and six deaths occurred in the transplanted patients. Two of these deaths were caused by tumor recurrence. The other deaths were caused by sepsis, or chronic rejection and subsequent graft failure resulting from hepatic artery thrombosis. Complications of transhepatic catheter placement for brachytherapy included bile duct perforation (four patients) and biliaryportal vein fistula (one patient) [33]. The Mayo clinic also reported a series of 148 patients affected by unresectable stage I or II CCA of the hepatic bifurcation initially receiving external-beam irradiation plus 5-FU, followed by brachytherapy with iridium and concomitant protracted venous infusion of 5-FU until OLT. After irradiation, patients underwent an exploratory laparotomy to exclude metastatic disease. 90 out of 148 patients have completed neoadjuvant treatment and underwent to OLT and the 5-year survival rate in the group of transplanted patients was $71 \%$. The good results with this protocol were related to several factors: the external and internal radiation therapy (useful for controlling wall and perineural invasion); the strict patient selection, as all were stages I-II, unlike other series in which stages III-IV exceed $40 \%$, and most of those enrolled patients were young. The neoadjuvant treatment was so effective that no tumor was found in the explanted liver (even though cytology prior to LT had been positive). Unfavorable prognostic factors in those series were: age $>45$ years, carbohydrate antigen 19-9 > 100, previous cholecystectomy, residual tumor of $>2 \mathrm{~cm}$, perineural invasion, and waiting time $>100$ days [34-36]. Moreover, another multicenter analysis pooled the experiences of 12 transplant centers that had, from 1993 to 2010, collectively treated 287 patients under similar 
protocols [37]. Five-year recurrence free survival in an intent-to-treat analysis was $53 \%$, while posttransplant 5 -year survival was $65 \%$, highlighting the necessity for careful patient selection.

Another retrospective study evaluated the activity of a neoadjuvant chemoradiotherapy (SBRT) followed by capecitabine until OLT in 17 PH-CCA patients. During neoadjuvant therapy, there were a total of 35 adverse events and capecitabine dose reductions were required on five occasions. Ultimately, six patients received OLT, 11 transplant-related complications occurred, and 1-year survival after transplant was $83 \%$ [38].

Those retrospective and prospective data underlined the role and feasibilirty of different neoadjuvant chemoradiation protocols combined with OLT in a very selected group of CCA patients (a good prognosis PH-CCA patients without a clear possibility of an upfront surgery) but no randomized trials has been conducted for comparing this type of integrated treatment to the only surgery, or to the only OLT.

\section{- Neoadjuvant treatment of distal cholangiocarcinoma}

The term extrahepatic cholangiocarcinoma (EH-CCA) refers to a group of neoplasms originating distally to the second order bile branches of the right and left hepatic ducts and they are then divided into PH-CCA and distal bile duct's carcinoma (or distal CCA). While locally advanced $\mathrm{PH}-\mathrm{CCA}$ can receive different type of multimodal treatments in order to reach the R0 resection, distal CCA are usually treated with Whipple procedure (pancreaticoduodenectomy) including pylorus preserving coupled with porta hepatis lymphadenectomy while segmental bile duct resection with locoregional lymph nodes excision it is not yet universally accepted [39]. Therefore, criteria of inoperability are the same of pancreatic cancer $[40,41]$.

Neoadjuvant treatments could represent a challenging strategy of management of distal CCA also considering that the ESPAC-3 trial failed in demonstrating the survival benefit of adjuvant chemotherapy in patients affected by resected periampullary carcinomas [42]. Up to date, there is still not consensus about preoperative treatment of distal-CCA due to lack of studies exploring the efficacy of this strategy probably because of the rarity of this pathology and the absence of a proper selection of patients. The current scientific evidences derived from single institution experiences and the majority of these are retrospective studies and not randomized trial. The Glazer's retrospective analysis [15] included different type of CCA (both the EH-CCA and distal-CCA) or GBC even if it is well known that division in anatomic sites correlate with different gene expression profile, different clinical behavior and sensitivity to citotoxic agents $[1,8,18]$. Mc Master and colleagues [43] reported the positive experience of preoperative chemoradiation for extrahepatic cholangiocarcinoma in their institution from 1963 to 1996. Nine (five perihilar and four distal cholangiocarcinoma) of 51 patients with unresectable disease underwent preoperative chemoradiation using continue infusion of 5-FU as a radiation sensitizer. After that, surgery was performed; two patients with perihilar tumors received an additional 10 Gy boost of irradiation delivered with brachytherapy and three of the four patients with distal tumors received an external beam intraoperative radiation therapy (IORT) to the area of the celiac axis and retroperitoneum. Three of nine patients that underwent chemoradiation experienced complete pathological response (two patients with perhylar and one with distal tumor). More recently, another single center retrospective study [44] analyzed the role of gemcitabine as neoadjuvant therapy in 22 patients with unresectable BTC. Eight of 22 patients were able to undergo surgery after the cytotoxic treatment (four of them where R0). Patients who underwent surgery had significantly benefit in survival. Another trial confirmed promising efficacy from chemoradiotherapy; 106 patients underwent 5-FU- or gemcitabine-based concurrent chemoradiotherapy for locally advanced BTC (including distal CCA) and 70 patients where randomized to best supportive care. The median overall survival was 42.57 weeks $(95 \%$ CI, 35.85-49.30) versus 13.29 weeks (95\% CI $10.42-16.15)$ in the combined therapy and best supportive care group, respectively [45].

\section{Conclusions \& future perspectives}

Despite significant advances in the optimization and surgical management of patients with BTC, the only chance for long-term survival still remains surgical resection with negative pathological margins (at least $1 \mathrm{~cm}$ ) while liver transplantation may be brought to a small selected group of patients [46]. Neoadjuvant approach is still an attractive field of research since it could facilitate tumor shrinkage and downstaging. Nevertheless, the aggressive nature of disease, together with 
modest efficacy of standard chemotherapy or new biological agents, result in slow or insignificant progress of this setting. Large prospective trials are lacking and only small retrospective series are currently available with contrasting conclusions. A special effort should be made to amass patients with clinical T3/T4/N1 disease on clinical trial or registry paying attention to a proper stratification (e.g., by site, performance status, node involvement, primary tumor dimension, ECOG PS) and an accurate stadiation before starting neoadjuvant therapy. In this sense, chemoradiotherapy followed by OLT seems to be the most promising strategy in intrahepatic/perihilar diseases with high curative rate. But it also appeared clear that this approach is burdened by high mobility and mortality when selection of patients is underpowered and the pretransplantation treatment protocol have to be standardized (external-beam irradiation combined with brachytherapy vs SBRT). Moreover, new types of local ablative treatments (such as radioembolization and SBRT) with or without chemotherapy need to be prospectively studied as preoperative therapy of IH-CCA in larger multicenter trials. Future trials could also investigate the activity of neoadjuvant protocol based on new polichemotherapy schedules which have already demonstrated their activity as conversion therapy for pancreatic cancer (such as FOLIFIRNOX or Gemcitabine and S1) $[47,48]$.

Financial \& competing interests disclosure The authors have no relevant affiliations or financial involvement with any organization or entity with a financial interest in or financial conflict with the subject matter or materials discussed in the manuscript. This includes employment, consultancies, honoraria, stock ownership or options, expert testimony, grants or patents received or pending, or royalties. No writing assistance was utilized in the production of this manuscript.

\section{Open access}

This work is licensed under the AttributionNonCommercial-NoDerivatives 4.0 Unported License. To view a copy of this license, visit http://creativecommons.org/ licenses/by-nc-nd/4.0.

\section{References}

Papers of special note have been highlighted as:

- of interest; $\bullet \bullet$ of considerable interest

1 Sia D, Tovar V, Moeini A et al. Intrahepatic cholangiocarcinoma: pathogenesis and rationale for molecular therapies. Oncogene 32 (41), 4861-4870 (2013).

2 Bragazzi MC, Cardinale V, Carpino G et al. Cholangiocarcinoma: epidemiology and risk factors. Transl. Gastrointest. Cancer 1, 21-32 (2012).

3 Shaib YH, Davila JA, McGlynn K et al. Rising incidence of intrahepatic cholangiocarcinoma in the United States: a true increase? J. Hepatol. 40 (3), 472-477 (2004).

4 Poultsides GA, Zhu AX, Choti MA et al. Intrahepatic cholangiocarcinoma. Surg. Clin. N. Am. 90 (4), 817-837 (2010).

5 Patel T. Increasing incidence and mortality of primary intrahepatic cholangiocarcinoma in the United States. Hepatology 33 (6), 1353-1357 (2001).

6 Misra S, Chaturvedi A, Misra NC et al. Carcinoma of the gall bladder. Lancet Oncol. 4(3), 167-176 (2003).

7 Levi F, Lucchini F, Negri E et al. The recent decline in gall bladder cancer mortality in Europe. Eur. J. Cancer Prev. 12(4), 265-267 (2003).
8 Blechacz B, Gores GJ Cholangiocarcinoma: advances in pathogenesis, diagnosis, and treatment. Hepatology 48 (1), 308-321 (2008).

9 Grendar J, Grendarova P, Sinha R et al. Neoadjuvant therapy for downstaging of locally advanced hilar cholangiocarcinoma: a systematic review. HPB (Oxford) 16(4), 297-303 (2014).

10 Witzigmann H, Berr F, Ringel U et al. Surgical and palliative management and outcome in 184 patients with hilar cholangiocarcinoma: palliative photodynamic therapy plus stenting is comparable to $\mathrm{r} 1 / \mathrm{r} 2$ resection. Ann. Surg. 244 (2), 230-239 (2006).

11 Jarnagin WR. Biliary tract: is there a role for neoadjuvant and adjuvant therapy in biliary cancer? Nat. Rev. Gastroenterol. Hepatol. 9, 622-623 (2012).

12 Nccn Guidelines Hepatobiliary Cancers, Version 2. www.nccn.org (2015).

13 de Aretxabala X, Losada H, Mora J et al. Neoadjuvant chemoradiotherapy in gall bladder cancer. Rev. Med. Chil. 132 (1), 51-57 (2004).

14 Engineer R, Wadasadawala T, Mehta S et al. Chemoradiation for unresectable gall bladder cancer: time to review historic nihilism? J. Gastrointest. Cancer 42 (4), 222-227 (2011).
- A small report showing the activity of gemcitabine-based chemotherapy as neoadjuvant treatment of gall bladder cancer (GBC).

15 Glazer ES, Liu P, Abdalla EK et al. Neither neoadjuvant nor adjuvant therapy increases survival after biliary tract cancer resection with wide negative margins. J. Gastrointest. Surg. 16 (9), 1666-1671 (2012).

16 Sirohi B, Mitra A, Jagannath P et al. Neoadjuvant chemotherapy in patients with locally advanced gall bladder cancer. Future Oncol. 11(10), 1501-1509 (2015).

- A retrospective analysis showing the activity of gemcitabine-platinum-based chemotherapy as neoadjuvant treatment of GBC.

17 Blechacz B, Komuta M, Roskams T et al. Clinical diagnosis and staging of cholangiocarcinoma. Nat. Rev. Gastroenterol. Hepatol. 8 (9), 512-522 (2011).

18 Jarnagin WR, Klimstra DS, Hezel M et al. Differential cell cycle-regulatory protein expression in biliary tract adenocarcinoma: correlation with anatomic site, pathologic variables, and clinical outcome. J. Clin. Oncol. 24 (7), 1152-1160 (2006).

- A clinicopathologic study showing the different biologic characteristics of biliary tract cancer (BTC) by anatomic site.

19 Kim JH, Won HJ, Shin YM et al. 
Radiofrequency ablation for the treatment of primary intrahepatic cholangiocarcinoma. AJR Am. J. Roentgenol. 196 (2), W205-W209 (2011).

20 Carrafiello G, Laganà D, Cotta E et al. Radiofrequency ablation of intrahepatic cholangiocarcinoma: preliminary experience. Cardiovasc. Intervent. Radiol. 33 (4), 835-839 (2010).

21 Fu Y, Yang W, Wu W et al. Radiofrequency ablation in the management of unresectable intrahepatic cholangiocarcinoma. J. Vasc. Interv. Radiol. 23 (5), 642-649 (2012).

22 Valle J, Wasan H, Palmer DH et al. Cisplatin plus gemcitabine versus gemcitabine for biliary tract cancer. N. Engl. J. Med. 362 (14), 1273-1281 (2010).

23 Park SY, Kim JH, Yoon HJ et al. Transarterial chemoembolization versus supportive therapy in the palliative treatment of unresectable intrahepatic cholangiocarcinoma. Clin. Radiol. 66 (4), 322-328 (2011).

24 Saxena A, Bester L, Chua TC et al. Yttrium-90 radiotherapy for unresectable intrahepatic cholangiocarcinoma: a preliminary assessment of this novel treatment option. Ann. Surg. Oncol. 17 (2), 484-491 (2010).

25 Mouli S, Memon K, Baker T et al. Yttrium-90 Radioembolization for Intrahepatic Cholangiocarcinoma: Safety, Response, and Survival Analysis. J. Vasc. Interv. Radiol. 24(8), 1227-1234 (2013).

- A pilot study showing the activity radioembolization as neoadjuvant treatment of intrahepatic cholangiocarcinoma (IH-CCA).

26 Al-Adra DP, Gill RS, Axford SJ et al. Treatment of unresectable intrahepatic cholangiocarcinoma with yttrium-90 radioembolization: a systematic review and pooled analysis. Eur. J. Surg. Oncol. 41 (1), 120-127 (2015).

- A systematic review showing the activity radioembolization as neoadjuvant treatment of IH-CCA.

27 Servajean C, Gilabert M, Piana G et al. One case of intrahepatic cholangiocarcinoma amenable to resection after radioembolization. World J. Gastroenterol. 20(17), 5131-5134 (2014).

28 Rayar M, Sulpice L, Edeline J et al. Intra-arterial Yttrium-90 radioembolization combined with systemic chemotherapy is a promising method for downstaging unresectable huge intrahepatic cholangiocarcinoma to surgical treatment. Ann. Surg. Oncol. 22(9), 3102-3108. (2015).
29 Martinez BK, Flanders V, Gupta NK et al. Development of a Y90 radioembolization program in a community hospital setting for treatment of metastatic and primary liver tumors. J. Vasc. Interv. Radiol. 24(5), 759. e32-759.e33 (2013).

30 Ibarra RA, Rojas D, Snyder L et al. Multicenter results of stereotactic body radiotherapy (SBRT) for non-resectable primary liver tumors. Acta Oncol. 51(5), 575-583 (2012).

- A combined multicentre database showing the activity SBRT in treatment of IH-CCA.

31 Busuttil RW, Farmer DG. The surgical treatment of primary hepatobiliary malignancy. Liver Transpl. Surg. 2(5), 114-130 (1996).

32 Meyer CG, Penn I, James L. Liver transplantation for cholangiocarcinoma: results in 207 patients. Transplantation 69 (8), 1633-1637 (2000).

33 Sudan D, DeRoover A, Chinnakotla S et al. Radiochemotherapy and transplantation allow long- term survival for nonresectable hilar cholangiocarcinoma. Am. J. Transplant. 2 (8), 774-779 (2002).

34 De Vreede I, Steers JL, Burch PA et al. Prolonged disease-free survival after orthotopic liver transplantation plus adjuvant chemoirradiation for cholangiocarcinoma. Liver Transpl. 6 (3), 309-316 (2000).

- The Mayo Clinic experience about neoadjuvant chemoradiotherapy and orthotopic liver transplantation (OLT) for treatment of CCA.

35 Rosen CB, Heimbach JK, Gores GJ. Surgery for cholangiocarcinoma: the role of liver transplantation. HPB (Oxford) 10 (3), 186-189 (2008).

36 Heimbach JK, Gores GJ, Haddock MG et al. Predictors of disease recurrence following neoadjuvant chemoradiotherapy and liver transplantation for unresectable perihilar cholangiocarcinoma. Transplantation 82 (12), 1703-1707 (2006).

37 Rea DJ, Heimbach JK, Rosen CB et al. Liver transplantation with neoadjuvant chemoradiation is more effective than resection for hilar cholangiocarcinoma Ann. Surg. 242(3), 451-458; discussion 458-461 (2005).

- The Mayo Clinic experience showing that neoadjuvant chemoradiotherapy and OLT was more effective than resection for perihilar (PH)-CCA.

38 Welling TH, Feng M, Wan S et al. Neoadjuvant stereotactic body radiation therapy, capecitabine, and liver transplantation for unresectable hilar cholangiocarcinoma. Liver Transpl. 20 (1), 81-88 (2014).

- A pilot study showing the feasibility of neoadjuvant SBRT combined with chemotherapy and OLT for PH-CCA.

39 Akamatsu N, Sugawara Y, Hashimoto D. Surgical strategy for bile duct cancer: Advances and current limitations World J. Clin. Oncol. 2(2), 94-107 (2011).

40 Ghouri YA, Mian I, Blechacz B. Cancer review: cholangiocarcinoma. J. Carcinog. 14, 1 (2015).

41 Nccn Guidelines Pancreatic Adenocarcinoma, Version 2. www.nccn.org (2015).

42 Neoptolemos JP, Moore MJ, Cox TF et al. Effect of adjuvant chemotherapy with fluorouracil plus folinic acid or gemcitabine vs observation on survival in patients with resected periampullary adenocarcinoma: the espac-3 periampullary cancer randomized trial. JAMA 308 (2), 147-156 (2012).

43 McMasters KM, Tuttle TM, Leach SD et al. Neoadjuvant chemoradiation for extrahepatic cholangiocarcinoma. Am. J. Surg. 174 (6), 605-608 (1997).

- A retrospective study showing the activity of chemoradiotherapy as neoadjuvant treatment for extrahepatic-CCA.

44 Kato A, Shimizu H, Ohtsuka M et al. Surgical resection after downsizing chemotherapy for initially unresectable locally advanced biliary tract cancer: a retrospective single-center study. Ann. Surg. Oncol. 20 (1), 318-324 (2013).

45 Yi SW, Kang DR, Kim KS. Efficacy of concurrent chemoradiotherapy with 5fluorouracil or gemcitabine in locally advanced biliary tract cancer. Cancer Chemother. Pharmacol. 73(1), 191-198 (2014).

46 Berdah SV, Delpero JR, Garcia S et al. A western surgical experience of peripheral cholangiocarcinoma. Br. J. Surg. 83 (11), 1517-1521 (1996).

47 Motoi F, Ishida K, Fujishima F et al. Neoadjuvant chemotherapy with gemcitabine and $S-1$ for Resectable and Borderline Pancreatic Ductal Adenocarcinoma: results from a prospective multi-institutional Phase 2 Trial. Ann. Surg. Oncol. 20 (12), 3794-3801 (2013).

48 Petrelli F, Coinu A, Borgonovo K et al. FOLFIRINOX-based neoadjuvant therapy in borderline resectable or unresectable pancreatic cancer: a meta-analytical review of published studies. Pancreas 44(4), 515-521 (2015). 